\title{
Conventional vs. High-definition tDCS: A Comparison of Neurophysiological and Behavioural Effects
}

\author{
Fabio Masina ( $\sim$ fabio.masina@ospedalesancamillo.net) \\ IRCCS San Camillo Hospital, Venice \\ Giorgio Arcara \\ IRCCS San Camillo Hospital, Venice \\ Eleonora Galletti \\ Department of General Psychology. University of Padua \\ Isabella Cinque \\ Department of General Psychology. University of Padua

\section{Luciano Gamberini} \\ Human Inspired Technology Research Centre. University of Padua

\section{Daniela Mapelli} \\ Human Inspired Technology Research Centre. University of Padua
}

\section{Research Article}

Keywords: High-definition tDCS, Transcranial direct current stimulation, Resting-state EEG, State-dependency, Motor cortex

Posted Date: December 18th, 2020

DOI: https://doi.org/10.21203/rs.3.rs-128899/v1

License: @ (1) This work is licensed under a Creative Commons Attribution 4.0 International License. Read Full License

Version of Record: A version of this preprint was published at Scientific Reports on April 7th, 2021. See the published version at https://doi.org/10.1038/s41598-021-87371-z. 


\section{Abstract}

High-definition transcranial direct current stimulation (HD-tDCS) seems to overcome a drawback of Conventional tDCS: the wide-spread diffusion of the electric field. Nevertheless, most of the differences that characterise the two techniques are based on mathematical simulations and not on real, behavioural and neurophysiological, data.

The study aims to compare Conventional and HD-tDCS, investigating differences both at a behavioural level, in terms of dexterity performance, and a neurophysiological level, as modifications of alpha and beta power as measured with EEG.

Thirty participants took part in three sessions, one for each montage: Conventional tDCS, HD-tDCS, and sham. In all the conditions, the anode was placed over $\mathrm{C} 4$, while the cathode/s placed according to the montage. At baseline, during, and after each stimulation condition, dexterity was assessed with a Finger Tapping Task. In addition, resting-state EEG was recorded at baseline and after the stimulation. Power spectrum density was calculated, selecting two frequency bands: alpha $(8-12 \mathrm{~Hz})$ and beta $(18-22 \mathrm{~Hz})$. Linear mixed effect models (LMMs) were used to analyse the modulation induced by tDCS. To evaluate differences among the montages and consider statedependency phenomenon, the post-stimulation measurements were covariate-adjusted for baseline levels.

We observed that HD-tDCS induced an alpha power reduction in participants with lower alpha. Conversely, Conventional tDCS induced a beta power reduction in participants with higher beta at baseline. Furthermore, data suggested a positive behavioural effect of HD-tDCS on dexterity in participants with lower beta at baseline.

Conventional and HD-tDCS distinctively modulated cortical activity. The study highlights the importance of considering state-dependency to determine the effects of tDCS on individuals.

\section{Introduction}

Transcranial direct current stimulation (tDCS) is a non-invasive brain stimulation (NIBS) technique that uses a constant weak electric current (generally 1-2 mA) to modulate specific brain areas over which it is applied ${ }^{1-3}$. The current passes through the scalp, skull, and cerebrospinal fluid down to the cortex, bringing membrane potential of neurons closer to or farther from the action potential threshold ${ }^{4}$. In Conventional tDCS, the current is delivered through two electrodes, an anode and a cathode, typically sized $25-35 \mathrm{~cm}^{2}$. One electrode is placed over the area of interest, while the other one is placed over another site, either cephalic or extracephalic 5 .

Despite the growing popularity of Conventional tDCS, especially as a promising tool for the treatment of clinical conditions, several criticisms have undermined the reliability of this technique: the inconsistency of tDCS effects and the difficulty to replicate previous findings ${ }^{6}$. The reasons behind the crisis of tDCS are related to several aspects, mostly methodological, which likely had a substantial impact in the replication of the results 7,8 .

A further critical aspect, partially neglected in the literature, is related to biophysical properties, and concerns the spatial distribution of the electric field produced by Conventional tDCS. As a matter of fact, Conventional tDCS produces a wide-spread electric field with low spatial specificity and peaks of intensity falling outside the active electrode 9,10 . Since the stimulation reaches brain areas that are structurally and functionally different, the electric field distribution may be another issue explaining such variability and inconsistency of tDCS-induced effects at both behavioural and neurophysiological levels.

Recently, new tDCS montages and devices have been introduced to overcome the limitation of Conventional tDCS. High-definition tDCS (HD-tDCS) montages use smaller electrodes than Conventional tDCS allowing the electric current to be delivered with increased density and focality. The most used HD-tDCS montage is the $4 \times 1$ ring configuration, which consists of one active electrode placed on the area of interest and four return electrodes on the surroundings. In this way, the delivered electric current is constrained and localised within the return electrodes ${ }^{9}$. Finite element method (FEM) models predict a different strength and distribution of electric field induced by Conventional and HD-tDCS ${ }^{10-12}$. In particular, specific electrode placements of HD-tDCS contribute to reduce the uncontrolled diffusion of tDCS-induced electric fields, thereby improving the spatial precision with which the electrical current can target specific cortical regions ${ }^{13}$. Findings coming from physiological studies have also presented a higher precision of HD-tDCS in modulating neurophysiological components as compared to Conventional tDCS 9,14 .

Although different electric field distributions have been found in computational studies concerning Conventional and HD-tDCS, no clear conclusion can be drawn on whether HD-tDCS can really produce a more focal and strong modulation than Conventional tDCS, both at 
behavioural and neurophysiological levels ${ }^{15,16}$. Indeed, most of the differences between the two techniques are based on mathematical simulations, and not on empirical effects of the stimulation.

In order to fill this gap and to further elucidate the differential effects of Conventional and HD-tDCS, the main goal of this research is to compare these two methods, investigating in the same study, for the first time, both behavioural and neurophysiological outcomes. Importantly, to evaluate tDCS effects, this study capitalises on some methodological advances as compared to common standards. The large majority of studies using tDCS investigate effects by comparing baseline and post-stimulation measurements ${ }^{17,18}$, or by using change scores, that is analysing effects obtained by subtracting baseline from post-stimulation measurements ${ }^{19,20}$. These widespread approaches assume several hidden statistical assumptions (often not satisfied) and ignore some well-known potential distortions. One of these is the phenomenon of regression to the mean ${ }^{21}$, according to which extreme values (very low or very high) tend to be closer to the mean in a repeated measure for pure statistical reasons. Moreover, baseline levels can constraint potential outcome of treatment also for other reasons. For example, if the behavioural scores are already at ceiling at baseline, no further improvement is possible and this cannot be explicitly taken into account using mean scores or change scores in the statistical analysis (as it happens with $t$-test or ANOVA).

To overcome these limitations and following gold standard for randomised control treatment design in biostatistics ${ }^{21}$, in the present study post-stimulation measurements were adjusted for baseline levels, considering these latter as covariates in statistical models. By using this approach, the question of the study changes from "Is HD-tDCS better than Conventional tDCS?" to "Are there specific conditions under which HD-tDCS is better than Conventional tDCS?". This is a methodological shift towards addressing more proper questions, as the investigation of tDCS effects cannot ignore the potential of observing actual modifications which highly depend on baseline values. Remarkably, applying this rationale and method to neural data (e.g., EEG data)allows to properly control for the initial activation state of brain before the stimulation, hence taking into account the phenomenon of state-dependency ${ }^{2,22}$. Although several articles advocate the importance of state-dependency in explaining the variability of observed effects or the failure of observing significant effects ${ }^{2,23}$, yet few studies actually take into account initial state as a variable in the analysis, so explicitly investigating the actual influence of statedependency effects.

In the present study, tDCS was used in combination with a motor task given the large body of evidence suggesting the technique would induce the strongest effects within the motor domain (for a review and meta-analysis, see ${ }^{24}$ ). In particular, tDCS was delivered with the aim to modulate participant's dexterity, which was assessed with a computerised version of the Finger Tapping Task, administered before, during, and after the stimulation stage. Furthermore, the Purdue Pegboard Test ${ }^{25}$ was used to assess dexterity in a non-computer based fashion. We expected that for the HD-tDCS condition we would observe a greater improvement in participants' dexterity relative to Conventional tDCS and sham. Furthermore, we expected greater dexterity improvement in Conventional tDCS relative to sham.

At a neurophysiological level, resting-state EEG was recorded before and after the stimulation stage. Power spectrum density was calculated selecting two EEG bands, respectively alpha $(8-12 \mathrm{~Hz})$ and beta band $(18-22 \mathrm{~Hz})$. The rationale of selecting alpha and beta bands is based on the functional role of these two bands. While the alpha frequency band has been linked with general inhibitory mechanisms ${ }^{26}$, thus liking the decrease of alpha power to motor facilitation, the beta power has been related to sensory-motor functions 27.

A recent review ${ }^{28}$ shows that anodal tDCS reduces alpha power while increasing beta power. For this reason, we expected that anodal HDtDCS would produce a greater modulation of the neurophysiological signal, leading to a significantly greater decrease of alpha and increase of beta power as compared to the Conventional and sham conditions. Moreover, we expected that Conventional tDCS would cause a greater modulation relative to sham.

\section{Results}

\section{The Finger Tapping Task}

The mean response times (RTs) and accuracy for each stimulation condition are summarised in Table 1.

With regard to RTs, in the model with the beta power entered as covariate, a main effect of stimulation condition was found $[F(2,29.9)=$ 3.51, $p=.043$ ]. Post-hoc contrasts did not show significant differences between the conditions (lowest $p=.113$ ). In this model, a significant stimulation condition * beta power at baseline stage interaction was found $[F(2,29.9)=3.58, p=.04]$, (Fig. 1). Contrasts were performed at the level of the 1 st, 2 nd, and 3rd quartile of the beta power at the baseline stage, respectively $-14.6,-14.3,-14$ power units. Post-hoc tests showed a tendency towards significance: for lower beta at baseline (i.e., -14.6 power units), HD-tDCS reduced RTs compared to sham 
(1514 ms vs. $1634 \mathrm{~ms}, p=.068$ ). No effect was identified in the models that considered as covariate the alpha power, as well as all the models on accuracy (see supplementary material).

\section{The Purdue Pegboard Test}

The mean performance at the Purdue Pegboard Test for each stimulation condition is shown in Table 1. The models considered in the analysis did not show any significant effects (see supplementary material).

Table 1

Mean and standard deviation (SD) of performance measures on the Finger Tapping Task and the Purdue Pegboard Test for Conventional tDCS, HD-tDCS, and sham.

\begin{tabular}{|c|c|c|c|c|c|c|c|c|c|}
\hline \multirow[t]{2}{*}{ Measures } & \multicolumn{3}{|c|}{ Conventional tDCS } & \multicolumn{3}{|l|}{ HD-tDCS } & \multicolumn{3}{|l|}{ Sham } \\
\hline & Baseline & Stimulation & $\begin{array}{l}\text { Post- } \\
\text { stimulation }\end{array}$ & Baseline & Stimulation & $\begin{array}{l}\text { Post- } \\
\text { stimulation }\end{array}$ & Baseline & Stimulation & $\begin{array}{l}\text { Post- } \\
\text { stimulation }\end{array}$ \\
\hline $\begin{array}{l}\text { FTT - RTs } \\
\text { (ms) }\end{array}$ & $\begin{array}{l}1956 \\
(810)\end{array}$ & 1745 (651) & $1582(615)$ & $\begin{array}{l}1996 \\
(798)\end{array}$ & 1792 (675) & 1582 (572) & $\begin{array}{l}2024 \\
(904)\end{array}$ & $1780(717)$ & 1663 (619) \\
\hline $\begin{array}{l}\text { FTT - } \\
\text { Accuracy } \\
(\%)\end{array}$ & $\begin{array}{l}87.9 \\
(7.7)\end{array}$ & $86.9(5.9)$ & $88(8.6)$ & $\begin{array}{l}87.6 \\
(9.1)\end{array}$ & $85.8(6.4)$ & $88.9(6)$ & $\begin{array}{l}87.8 \\
(8.1)\end{array}$ & $87.3(6.3)$ & $85.9(8.7)$ \\
\hline $\begin{array}{l}\text { PPT - } \\
\text { Left hand }\end{array}$ & - & - & $14.7(1.6)$ & - & - & $14.3(1.6)$ & - & - & $14.4(1.5)$ \\
\hline
\end{tabular}

\section{EEG analysis}

The model investigating the impact of tDCS on the alpha power showed several significant effects. F-tests showed a main effect of stimulation condition $[F(2,52.9)=9.69, p<.001]$, despite post-hoc contrasts did not show any significant differences among the conditions (lowest $p=.203$ ). In addition, a stimulation condition * alpha power baseline stage interaction was found $[F(2,52.9)=9.87, p<.001]$,

(Fig. 1). Contrasts were conducted at the level of the 1st, 2nd, and 3rd quartile of the alpha power at the baseline stage, respectively - 14, $-13.5,-13.2$ power units. Post-hoc tests showed that for lower power values of this covariate (i.e., -14 power units), HD-tDCS reduced the alpha power compared to sham $(-13.8$ vs. $-13.6, p=.013)$.

In the second model, in which the beta power was considered, analysis showed a main effect of stimulation condition $[F(2,80.5)=15.64, p$ $<.001$ ]. Post-hoc comparisons did not reveal a difference between the conditions (lowest $p=.408$ ). A significant interaction between stimulation condition * beta power baseline stage was found $[F(2,80.7)=15.45, p<.001]$, (Fig. 1$)$. By conducting contrasts at the 1 st, 2 nd, and 3rd quartile of the covariate, respectively - 14.6,-14.3,-13.9 power units, a reduction of beta power was found in Conventional tDCS compared to HD-tDCS (-14.2 vs. $-14, p=.002)$, and in Conventional tDCS compared to sham $(-14.2$ vs. $-14.1, p=.045)$, for higher beta power values at the baseline stage (i.e., -13.9 power units).

\section{Discussion}

The present study aims to provide new evidence characterising functional differences between Conventional and HD-tDCS. To properly evaluate effects of both the montages, we adopted a state-of-the-art approach for the statistical analysis, that overcomes some common suboptimal statistical methods and focalises on properly identifying the conditions associated with specific treatment outcome ${ }^{21}$. This approach consisted in adjusting the post-stimulation measurements for baseline levels, considering these latter as covariates in the analysis. Of importance, this method allowed to properly account for state-dependency $2,29,30$, a phenomenon often evoked to explain variability and inconsistency of tDCS-induced effects ${ }^{31}$, but generally neglected in statistical analysis.

Results from the present study outline distinctive modulation of Conventional and HD-tDCS. This evidence, observed empirically, is possibly related to the different strength and distribution of the electric field induced by Conventional and HD-tDCS, as predicted by FEM computational models ${ }^{10-12}$. Firstly, we found that following HD-tDCS, a reduction of EEG alpha power during resting state could be 
observed. This result is consistent with previous studies showing an inhibitory effect of anodal tDCS on alpha power ${ }^{28}$. The functional role of alpha is commonly related to cortical deactivation and inhibition $26,32,33$ and animal models provide evidence that alpha-band oscillations have an inhibitory influence on the generation of spikes ${ }^{34}$. Consequently, if an increase of alpha power reflects inhibition, a reduction in power should reflect release from inhibition, supporting evidence that anodal HD-tDCS on M1 induces cortical excitability ${ }^{14}$.

In line with our expectations, the state of neural activation before applying HD-tDCS played a role in the modulation of alpha. Specifically, a reduction of alpha power was observed only in participants that had lower EEG alpha before HD-tDCS. A possible explanation of this result may rely on the cognitive role linked to alpha band, namely its involvement in attention ${ }^{26}$. This viewpoint would suggest that only participants with a proper attentional asset (i.e., lower alpha and, consequently, higher level of attention before the stimulation) benefited from a release of inhibition following the administration of HD-tDCS. Remarkably, this result contributes to identify which physiological markers can predict tDCS effects, especially the effects of HD-tDCS that is relatively recent among NIBS techniques.

Contrary to our expectations, we found that Conventional tDCS induced a reduction of beta power. Specifically, in our study the reduction of beta occurred for participants who already showed a higher level of beta power before the administration of Conventional tDCS. The inhibition of beta induced by anodal tDCS is not unusual in the literature ${ }^{35,36}$, despite previous evidence showing an opposite pattern, namely an increase of beta power ${ }^{28}$.

The inconsistency of these findings may be accounted for as a consequence of the uncontrolled spread of the electric field in Conventional tDCS $^{9}$. As shown, in tDCS the current generally concentrates at the edge of the electrode ${ }^{37}$. Thus, the larger is the electrode size the lower is the probability to keep the edge of the electrode over the target area. With the unlikelihood of being able to control the electric current diffusion, a possible concern arises in the fact that tCDS may affect more than just the target region. Consequently, Conventional tDCS, which typically uses large electrodes sized $25-35 \mathrm{~cm}^{2}$, may lead to undesired or mixed effects due to the stimulation of nearby areas connected to the target area. Further studies are necessary to confirm this hypothesis, possibly by comparing functional outcomes of Conventional and HD-tDCS in the same study.

With respect to behavioural outcomes, HD-tDCS produced some evidence of motor improvement in participants who had lower beta power at the baseline stage. This finding would suggest that HD-tDCS can induce enhancement of unimanual dexterity, as shown by a previous study ${ }^{38}$. Functionally, beta power has been hypothesised to be linked to sensory-motor functions ${ }^{27}$. Generally, a reduction of beta power is seen in planning and execution of motor action, followed by an increase of power after the end of the movement ${ }^{39,40}$. However, the dynamic fluctuations of beta power before, during, and after a voluntary movement have to reflect several underlying processes. While the desynchronisation of beta (i.e., power decrease) would be related to the asynchronous activation of the motor cortex during a movement, the synchronization (i.e., power increase) seems to reflect several mechanisms including motor control ${ }^{41}$ and the maintenance of tonic activity at the cost of voluntary movements ${ }^{42}$. Interestingly, this latter hypothesis is coherent with a recent study showing that the increase of beta activity would induce a slowdown of movements ${ }^{43}$. Thus, the improvement in dexterity granted by HD-tDCS might prove to be higher if the brain state has an adequate level of disposition to the movement onset (i.e., lower beta power). Nevertheless, given the small effect found, further research is necessary to confirm the possibility to enhance dexterity by HD-tDCS, possibly adopting a different task or investigating clinical population, in which the risk of a ceiling effect is reduced and the potential to improve motor performance is higher 44 .

Of importance, from a statistical point of view, results of this study underline the importance of adjusting the post-stimulation measurements for baseline levels, including these latter as covariates in statistical models. This approach, in line with recent suggestions for randomised control treatment designs in biostatistics ${ }^{21}$, should be considered as a good practice in future NIBS studies, especially with the aim to investigate the effect of stimulation not only per se but also in relation to the initial state of brain, and to the actual potential to be influenced by tDCS.

In summary, the present study contributes to characterise the differences between Conventional and HD-tDCS, both from a behavioural and neurophysiological perspective. HD-tDCS represents a recent advance in NIBS since it would overcome the low precision of Conventional tDCS. However, few studies have compared whether the increased focality of HD-tDCS could determine different modulation. Interestingly, our findings support this theory and show how HD-tDCS can induce more predictable outcomes than Conventional tDCS. Significantly, the present study also highlights the importance of considering the initial state of brain activity before tDCS application, as it can be crucial to influence the effects of tDCS.

\section{Methods And Materials}




\section{Participants}

Thirty participants were recruited from the University of Padua. They were matched for gender (15 males and 15 females) and their age range was between $19-30$ years old (mean age $=23.4$, standard deviation $(S D)=1.9$; mean education $=16.2, S D=1.3$ ). All participants were right-handed, as indicated by the Edinburgh Handedness Inventory ${ }^{45}$ (mean laterality score: $80.5, S D$ : 16.8), and reported normal or corrected-to-normal visual acuity.

Participants with a history of neurological or psychiatric diseases were excluded from the study. They were all checked for tDCS exclusion criteria ${ }^{46}$. All safety procedures were in line with tDCS guidelines ${ }^{46}$. Before the experiment, participants gave their written informed consent. The study was approved by the ethics committee of the Human Inspired Technology (HIT) Research Centre in Padua (nr. 2019_39) and was compliant with the ethical principles of the 1964 Declaration of Helsinki.

\section{Transcranial direct current stimulation (tDCS)}

The tDCS and EEG recordings were carried out through a multi-focal tDCS-EEG device (StarStim, Barcelona) with 20 channels. The system was remotely controlled via the Neuroelectrics Instrument Controller (NIC; v2.0.11.4). Participants were involved in three experimental sessions, during which a different tDCS montage was applied, namely Conventional tDCS or HD-tDCS.

In Conventional tDCS, two circular saline-soaked surface sponge electrodes (surface $=25 \mathrm{~cm}^{2}$; current density: $0.06 \mathrm{~mA} / \mathrm{cm}^{2}$ ) were used. The anode (active electrode) was placed on C4 (International 10-20 EEG System), while the cathode (return electrode) was placed over the contralateral (left) shoulder of participants.

In HD-tDCS, a $4 \times 1$-ring configuration with sintered Ag/AgCl electrodes (surface $=3.14 \mathrm{~cm}^{2}$; current density: $0.48 \mathrm{~mA} / \mathrm{cm}^{2}$ ) was adopted. The anode was placed over $\mathrm{C} 4$ and the four cathodes on FC2, FC6, CP2, CP6. In this electrode configuration, the electric current is assumed to be less spread than Conventional tDCS 9,14,47, as shown in electric field models (Fig. 2) simulated with SimNIBS ${ }^{48}$.

Along with the real tDCS, a sham condition was included where the montages were counterbalanced. Hence, $50 \%$ of participants received sham with the Conventional tDCS montage and the other $50 \%$ received sham with the HD-tDCS montage.

At the end of each session, participants completed a questionnaire of tDCS-related sensations ${ }^{49}$. Remarkably, participants were not able to distinguish between real tDCS and sham [session 1: Wald $\chi^{2}(2)=.37, p=.83$; session 2: Wald $\chi^{2}(2)=1.25, p=.53$; session 3 : Wald $\chi^{2}(2)$ $=.1 .88, p=.392]$.

\section{EEG recording}

The EEG signal was recorded with $20 \mathrm{Ag} / \mathrm{AgCl}$ sintered electrodes (StarStim, Barcellona) mounted on an elastic cap according to the International 10-20 system. The EEG electrodes were placed on the following sites: Fp1, Fp2, F7, F3, Fz, F4, F8, C3, Cz, C4, T7, T8, P7, P3, P4, P8, 01, 02 (Fig. 3). All recordings were referenced to the Pz electrode, while the ground electrode was placed on Oz. Raw data were digitalised with a frequency of $500 \mathrm{~Hz}$.

\section{Tasks}

The description of the behavioural tasks performed by participants (i.e., the Finger Tapping Task and the Purdue Pegboard Test) is available in supplementary material. The Fig. 3 shows a representation the Finger Tapping Task.

\section{Procedure}

Participants were involved in three experimental sessions (Conventional tDCS, HD-tDCS, and sham), carried out on separate days and separated by a washout period lasting between 6 and 16 days. Importantly, the stimulation conditions were counterbalanced within the three experimental sessions. Each experimental session was divided into six steps.

Firstly, the EEG headcap was placed on the scalp. All impedances were kept below $5 \mathrm{~K} \Omega$. The session started with 5 minutes of restingstate EEG. During the EEG recording, participants were asked to stare a fixation point kept at $60 \mathrm{~cm}$ distance. Successively, participants performed the Finger Tapping Task (baseline stage) without any EEG recording. After this stage, they were invited to perform the Finger Tapping Task for 20 minutes (stimulation stage) while being delivered the stimulation (Conventional tDCS, HD-tDCS, or sham). The stimulation condition lasted for 20 minutes, meaning that the real stimulation (i.e., Conventional tDCS or HD-tDCS) or sham was delivered for the entire duration of the Finger Tapping Task. Regardless of the stimulation condition, the current strength was $1.5 \mathrm{~mA}$ with a ramp

Page 6/13 
up/ramp down time of 30 seconds. In the sham condition, the current linearly increased for the first 30 seconds up to a $1.5 \mathrm{~mA}$ and then decreased to $0 \mathrm{~mA}$ in the next 30 seconds. After the stimulation stage, 5 minutes of resting-state EEG were recorded, following the aforementioned procedure. At the end of the EEG recording, participants performed the Finger Tapping Task for a third time (poststimulation stage). Finally, the Purdue Pegboard Test was administered. Figure 3 shows a representation of the procedure.

\section{Statistical analysis}

All data were analysed using RStudio software ${ }^{50}$ (version 1.2) and packages Ime $4{ }^{51}$, ImerTest ${ }^{52}$, car ${ }^{53}$, and emmeans ${ }^{54}$. Linear mixed effect models (LMMs) and generalised linear mixed effect models (GLMMs) were used. Significance of the fixed effects terms were assessed by means of $F$-test using Satterthwaite approximation ${ }^{55}$. Post-hoc pairwise contrasts were corrected with Tukey's multiple comparison test. For significant interactions between a continuous variable and a factor, estimated marginal means contrasts were performed at the level of the $1 \mathrm{st}, 2 \mathrm{nd}$, and 3rd quartile of the continuous variable. All relevant data and $\mathrm{R}$ scripts are available at https://osf.io/j4acs/, while all models performed in the analysis are available in supplementary material.

In the models, post-stimulation measurements, both behavioural and neurophysiological, were covariate-adjusted for baseline levels ${ }^{21,56}$.

\section{The Finger Tapping Task}

Participant's performance at the Finger Tapping Task was evaluated in terms of RTs and accuracy. RTs were measured as the time interval between string onset and the typing of the fifth digit (Fig. 3). RTs below $100 \mathrm{~ms}$ were removed from the analyses, as well as the strings not correctly typed.

Accuracy was computed as the ratio between the number of correct strings and the total number of strings. Error and correct strings were dichotomously coded, respectively as 0 and 1 . As a consequence, accuracy was analysed by using GLMMs for binomial data, using a logit link function ${ }^{57}$.

The models were fit to investigate the effects of tDCS on post-stimulation performance, considering baseline alpha or beta power as covariates. In the models, stimulation condition (Conventional tDCS, HD-tDCS, sham) and the covariates alpha/beta power at the baseline stage (see EEG analysis) were considered as fixed-effect factors, and participant, stimulation condition, and string repetition as random intercepts (Table A.1, supplementary material).

\section{The Purdue Pegboard Test}

Participants' performance at the Purdue Pegboard Test was scored as the mean number of pins, collars, and washers placed in the board, accordingly to the task instructions.

Since the anode was placed over $\mathrm{C} 4$ (right hemisphere), we restricted the analysis only to the mean score at the subtest performed with the left hand. Differences among the stimulation conditions (Conventional tDCS, HD-tDCS, sham) were investigated by fitting LMMs.

Stimulation condition (Conventional tDCS, HD-tDCS, sham) and the covariates alpha/beta power at the baseline stage were considered as the fixed effect factors, and participant was included as the random-effect (Table A.1, supplementary material).

\section{EEG analysis}

The EEG data were pre-processed offline with Brainstorm ${ }^{58}$ for Matlab R2017b (The Mathworks Natic, MA, USA). First, continuous EEG was band-pass filtered with a cut-off frequency of $0.1-47 \mathrm{~Hz}$. Then, the continuous EEG signal was visually inspected and channels with noise signal were removed. Independent component analysis ${ }^{59}$ was performed to correct the remaining artifacts (muscle activity and eye blinks). All independent components were visually inspected in terms of scalp distribution, frequency, timing and amplitude ${ }^{60}$. The mean number of removed independent components was $1.93(S D=.87)$. Afterwards, the EEG was segmented into 150 non-overlapping epochs of $2000 \mathrm{~ms}$.

Baseline correction was performed by subtracting the mean voltage of the whole epoch. The EEG signal was re-referenced to the mean of all channels. Epochs containing data points exceeding the amplitude of $-100 \mathrm{mV} /+100 \mathrm{mV}$ were excluded from the analysis. An average of $8.47 \%$ epochs were excluded. Successively, power spectrum density (Welch's method) was conducted to extract power [signal units/sqrt $(\mathrm{Hz}) * 10^{-5}$ ] relative to the two bands of interest: alpha band $(8-12 \mathrm{~Hz})$ and beta band $(18-22 \mathrm{~Hz})$. Data were averaged within 
each band and were log-transformed to reduce skewness. Only the power extracted from the electrodes close to the stimulation site (i.e., $\mathrm{F} 4, \mathrm{Cz}, \mathrm{T} 8, \mathrm{P} 4)$, and from C4 was considered in the analysis.

To investigate the effects of the stimulation conditions (Conventional tDCS, HD-tDCS, sham) on alpha/beta power at the post-stimulation stage, two LMMs were conducted. Stimulation condition (Conventional tDCS, HD-tDCS, sham) and the covariate alpha/beta power at the baseline stage were considered as fixed-effect factors. Random structure of the models consisted of participant and stimulation condition (Table A.1, supplementary material).

\section{Declarations DATA AVAILABILITY}

All relevant data and R scripts are available at https://osf.io/j4acs/, while the description of tasks (i.e., the Finger Tapping Task and the Purdue Pegboard Test) as well as all the models performed in the analysis are available in supplementary material.

\section{ACKNOWLEDGMENTS}

We would like to express our very great appreciation to Vincenza Tarantino for her valuable and constructive suggestions during the writing of this study. Her willingness to give her time so generously has been very much appreciated.

\section{AUTHOR CONTRIBUTIONS}

FM: Conceptualization, Investigation, Methodology, Data curation, Formal analysis, Visualization, Writing - original draft, Validation; GA: Conceptualization, Supervision, Methodology, Formal analysis, Visualization, Writing - original draft, Validation; EG: Investigation, Formal analysis, Writing - original draft; IC: Investigation, Formal analysis, Writing - original draft; LG: Conceptualization, Supervision, Validation; DM: Conceptualization, Supervision, Validation.

\section{ADDITIONAL INFORMATION}

Declaration of competing interest: all authors have no conflicts to report.

\section{FUNDING}

This research did not receive any specific grant from funding agencies in the public, commercial, or not-for-profit sectors.

\section{References}

1. Bikson, M. et al. Safety of Transcranial Direct Current Stimulation: Evidence Based Update 2016. Brain Stimulation (2016) doi:10.1016/j.brs.2016.06.004.

2. Fertonani, A. \& Miniussi, C. Transcranial electrical stimulation: What we know and do not know about mechanisms. Neuroscientist 23, 109-123 (2017)

3. Pellegrino, G. et al. Transcranial direct current stimulation over the sensory-motor regions inhibits gamma synchrony. Hum. Brain Mapp. (2019) doi:10.1002/hbm.24556.

4. Liu, A. et al. Immediate neurophysiological effects of transcranial electrical stimulation. Nature Communications (2018) doi:10.1038/s41467-018-07233-7.

5. Woods, A. J. et al. A technical guide to tDCS, and related non-invasive brain stimulation tools. Clinical Neurophysiology vol. 127 10311048 (2016).

6. Filmer, H. L., Mattingley, J. B. \& Dux, P. E. Modulating brain activity and behaviour with tDCS: Rumours of its death have been greatly exaggerated. Cortex (2020) doi:10.1016/j.cortex.2019.10.006.

7. Horvath, J. C., Carter, O. \& Forte, J. D. Transcranial direct current stimulation: Five important issues we aren't discussing (but probably should be). Frontiers in Systems Neuroscience (2014) doi:10.3389/fnsys.2014.00002. 
8. Parkin, B. L., Ekhtiari, H. \& Walsh, V. F. Non-invasive Human Brain Stimulation in Cognitive Neuroscience: A Primer. Neuron (2015) doi:10.1016/j.neuron.2015.07.032.

9. Edwards, D. et al. Physiological and modeling evidence for focal transcranial electrical brain stimulation in humans: A basis for highdefinition tDCS. Neuroimage (2013) doi:10.1016/j.neuroimage.2013.01.042.

10. Datta, A. et al. Gyri-precise head model of transcranial direct current stimulation: Improved spatial focality using a ring electrode versus conventional rectangular pad. Brain Stimul. 2, (2009).

11. Alam, M., Truong, D. Q., Khadka, N. \& Bikson, M. Spatial and polarity precision of concentric high-definition transcranial direct current stimulation (HD-tDCS). Phys. Med. Biol. (2016) doi:10.1088/0031-9155/61/12/4506.

12. Datta, A., Elwassif, M., Battaglia, F. \& Bikson, M. Transcranial current stimulation focality using disc and ring electrode configurations: FEM analysis. J. Neural Eng. (2008) doi:10.1088/1741-2560/5/2/007.

13. Hill, A. T., Rogasch, N. C., Fitzgerald, P. B. \& Hoy, K. E. Effects of prefrontal bipolar and high-definition transcranial direct current stimulation on cortical reactivity and working memory in healthy adults. Neuroimage(2017) doi:10.1016/j.neuroimage.2017.03.001.

14. Kuo, H. I. et al. Comparing cortical plasticity induced by conventional and high-definition $4 \times 1$ ring tDCS: A neurophysiological study. Brain Stimul. (2013) doi:10.1016/j.brs.2012.09.010.

15. Polanía, R., Nitsche, M. A. \& Ruff, C. C. Studying and modifying brain function with non-invasive brain stimulation. Nature Neuroscience vol. 21 174-187 (2018).

16. Morya, E. et al. Beyond the target area: an integrative view of tDCS-induced motor cortex modulation in patients and athletes. Journal of NeuroEngineering and Rehabilitation (2019) doi:10.1186/s12984-019-0581-1.

17. Alizadehgoradel, J. et al. Repeated stimulation of the dorsolateral-prefrontal cortex improves executive dysfunctions and craving in drug addiction: A randomized, double-blind, parallel-group study. Brain Stimul. (2020) doi:10.1016/j.brs.2019.12.028.

18. Bueno-Lopez, A., Eggert, T., Dorn, H. \& Danker-Hopfe, H. Slow oscillatory transcranial direct current stimulation (so-tDCS) during slow wave sleep has no effects on declarative memory in healthy young subjects. Brain Stimul. 12, 948-958 (2019).

19. Martens, G. et al. Randomized controlled trial of home-based 4-week tDCS in chronic minimally conscious state. Brain Stimul. (2018) doi:10.1016/j.brs.2018.04.021.

20. Khedr, E. M. et al. Effects of transcranial direct current stimulation on pain, mood and serum endorphin level in the treatment of fibromyalgia: A double blinded, randomized clinical trial. Brain Stimul. (2017) doi:10.1016/j.brs.2017.06.006.

21. Harrell, F. E. Regression modeling strategies: with applications to linear models, logistic and ordinal regression, and survival analysis. in (ed. Springer) 1-11 (2015). doi:10.1007/978-3-319-19425-7_1.

22. Silvanto, J. \& Pascual-Leone, A. State-dependency of transcranial magnetic stimulation. Brain Topography (2008) doi:10.1007/s10548-008-0067-0.

23. Silvanto, J., Bona, S. \& Cattaneo, Z. Initial activation state, stimulation intensity and timing of stimulation interact in producing behavioral effects of TMS. Neuroscience (2017) doi:10.1016/j.neuroscience.2017.09.002.

24. Hashemirad, F., Zoghi, M., Fitzgerald, P. B. \& Jaberzadeh, S. The effect of anodal transcranial direct current stimulation on motor sequence learning in healthy individuals: A systematic review and meta-analysis. Brain Cogn. (2016) doi:10.1016/j.bandc.2015.11.005.

25. Tiffin, J. \& Asher, E. J. The Purdue Pegboard: norms and studies of reliability and validity. J. Appl. Psychol. (1948) doi:10.1037/h0061266.

26. Klimesch, W. Alpha-band oscillations, attention, and controlled access to stored information. Trends in Cognitive Sciences (2012) doi:10.1016/j.tics.2012.10.007.

27. Betti, V., Della Penna, S., de Pasquale, F. \& Corbetta, M. Spontaneous Beta Band Rhythms in the Predictive Coding of Natural Stimuli. Neurosci. (2020) doi:10.1177/1073858420928988.

28. Annarumma, L., D’Atri, A., Alfonsi, V. \& De Gennaro, L. The efficacy of transcranial current stimulation techniques to modulate restingstate EEG, to affect vigilance and to promote sleepiness. Brain Sciences (2018) doi:10.3390/brainsci8070137.

29. Silvanto, J., Muggleton, N. \& Walsh, V. State-dependency in brain stimulation studies of perception and cognition. Trends Cogn. Sci. (2008) doi:10.1016/j.tics.2008.09.004.

30. Miniussi, C., Harris, J. A. \& Ruzzoli, M. Modelling non-invasive brain stimulation in cognitive neuroscience. Neuroscience and Biobehavioral Reviews vol. 37 1702-1712 (2013).

31. Horvath, J. C., Forte, J. D. \& Carter, O. Quantitative review finds no evidence of cognitive effects in healthy populations from singlesession transcranial direct current stimulation (tDCS). Brain Stimul. (2015).

Page $9 / 13$ 
32. Klimesch, W., Sauseng, P. \& Hanslmayr, S. EEG alpha oscillations: The inhibition-timing hypothesis. Brain Research Reviews (2007) doi:10.1016/j.brainresrev.2006.06.003.

33. Jensen, O. \& Mazaheri, A. Shaping functional architecture by oscillatory alpha activity: Gating by inhibition. Front. Hum. Neurosci. (2010) doi:10.3389/fnhum.2010.00186.

34. Haegens, S., Nácher, V., Luna, R., Romo, R. \& Jensen, O. a-Oscillations in the monkey sensorimotor network influence discrimination performance by rhythmical inhibition of neuronal spiking. Proc. Natl. Acad. Sci. U. S. A. (2011) doi:10.1073/pnas.1117190108.

35. Pellegrino, G. et al. Bilateral Transcranial Direct Current Stimulation Reshapes Resting-State Brain Networks: A Magnetoencephalography Assessment. Neural Plast. (2018) doi:10.1155/2018/2782804.

36. Goettker, A. et al. HD-tDCS over mIPS causally modulates online reach correction. bioRxiv (2019) doi:https://doi.org/10.1101/708693.

37. Faria, P., Hallett, M. \& Miranda, P. C. A finite element analysis of the effect of electrode area and inter-electrode distance on the spatial distribution of the current density in tDCS. J. Neural Eng. (2011) doi:10.1088/1741-2560/8/6/066017.

38. Pixa, N. H., Steinberg, F. \& Doppelmayr, M. High-definition transcranial direct current stimulation to both primary motor cortices improves unimanual and bimanual dexterity. Neurosci. Lett. (2017) doi:10.1016/j.neulet.2017.02.033.

39. Pfurtscheller, G., Stancák, A. \& Neuper, C. Post-movement beta synchronization. A correlate of an idling motor area? Electroencephalogr. Clin. Neurophysiol. (1996) doi:10.1016/0013-4694(95)00258-8.

40. Pfurtscheller, G. \& Lopes Da Silva, F. H. Event-related EEG/MEG synchronization and desynchronization: Basic principles. Clinical Neurophysiology (1999) doi:10.1016/S1388-2457(99)00141-8.

41. Murthy, V. N. \& Fetz, E. E. Coherent 25- To 35-Hz oscillations in the sensorimotor cortex of awake behaving monkeys. Proc. Natl. Acad. Sci. U. S. A. (1992) doi:10.1073/pnas.89.12.5670.

42. Baker, S. N., Kilner, J. M., Pinches, E. M. \& Lemon, R. N. The role of synchrony and oscillations in the motor output. in Experimental Brain Research (1999). doi:10.1007/s002210050825.

43. Khanna, P. \& Carmena, J. M. Beta band oscillations in motor cortex reflect neural population signals that delay movement onset. Elife (2017) doi:10.7554/eLife.24573.

44. Salazar, A. P. et al. Bi-cephalic transcranial direct current stimulation combined with functional electrical stimulation for upper-limb stroke rehabilitation: A double-blind randomized controlled trial. Ann. Phys. Rehabil. Med. 63, 4-11 (2020).

45. Oldfield, R. C. The assessment and analysis of handedness: The Edinburgh inventory. Neuropsychologia (1971) doi:10.1016/00283932(71)90067-4.

46. Antal, A. et al. Low intensity transcranial electric stimulation: Safety, ethical, legal regulatory and application guidelines. Clinical Neurophysiology (2017) doi:10.1016/j.clinph.2017.06.001.

47. Muthalib, M., Besson, P., Rothwell, J., Ward, T. \& Perrey, S. Effects of anodal high-definition transcranial direct current stimulation on bilateral sensorimotor cortex activation during sequential finger movements: An fnirs study. in Advances in Experimental Medicine and Biology (2016). doi:10.1007/978-1-4939-3023-4_44.

48. Thielscher, A., Antunes, A. \& Saturnino, G. B. Field modeling for transcranial magnetic stimulation: A useful tool to understand the physiological effects of TMS? in Proceedings of the Annual International Conference of the IEEE Engineering in Medicine and Biology Society, EMBS (2015). doi:10.1109/EMBC.2015.7318340.

49. Fertonani, A., Ferrari, C. \& Miniussi, C. What do you feel if I apply transcranial electric stimulation? Safety, sensations and secondary induced effects. Clin. Neurophysiol. (2015) doi:10.1016/j.clinph.2015.03.015.

50. Team, R. RStudio: Integrated development for R. (2019).

51. Bates, D., Maechler, M., Bolker, B. \& Walker, S. Ime4: Linear mixed-effects models using Eigen and S4. R package version 1.1-7, http://CRAN.R-project.org/package=Ime4. R Packag. version (2014).

52. Kuznetsova, A., Brockhoff, P. B. \& Christensen, R. H. B. ImerTest: Tests for random and fixed effects for linear mixed effect models. $R$ package version (2016).

53. Fox, J. \& Weisberg, S. An \{R\} Companion to Applied Regression, (top). Thousand Oaks CA: Sage. (2019).

54. Lenth, R., Singmann, H., Love, J., Buerkner, P. \& Herve, M. emmeans: Estimated Marginal Means, aka Least-Squares Means. R package version 1.15-15(2020) doi:10.1080/00031305.1980.10483031>.License.

55. Luke, S. G. Evaluating significance in linear mixed-effects models in R. Behav. Res. Methods (2017) doi:10.3758/s13428-016-0809-y.

56. Nunes, E. V. et al. Baseline matters: The importance of covariation for baseline severity in the analysis of clinical trials. Am. J. Drug Alcohol Abuse (2011) doi:10.3109/00952990.2011.596980.

Page 10/13 
57. Pinheiro, J. \& Bates, D. Linear and nonlinear mixed effects models (nIme). Package of the $R$ software for statistical computing on the CRAN repository. (2011).

58. Tadel, F., Baillet, S., Mosher, J. C., Pantazis, D. \& Leahy, R. M. Brainstorm: A user-friendly application for MEG/EEG analysis. Comput. Intell. Neurosci. (2011) doi:10.1155/2011/879716.

59. Makeig, S., Bell, A. J., Jung, T. \& Sejnowski, T. J. Independent Component Analysis of Electroencephalographic Data. in Advances in Neural Information Processing Systems 8 (1996).

60. Chaumon, M., Bishop, D. V. M. \& Busch, N. A. A practical guide to the selection of independent components of the electroencephalogram for artifact correction. J. Neurosci. Methods 250, 47-63 (2015).

\section{Figures}

\section{Figure 1}

Neurophysiological and behavioural results. Panel (a): Modulation of alpha power in the post-stimulation stage across stimulation conditions with alpha power at the baseline as covariate. In the $x$-axis, the $1 \mathrm{st}, 2 \mathrm{nd}$, and 3rd quartile of the covariate, respectively $-14,-13.5$, -13.2 power units, while in the $y$-axis, alpha power at the post-stimulation stage. At low levels of alpha power at the baseline (i.e., the 1 st quartile), results show a significant reduction of alpha power in the post-stimulation stage only for HD-tDCS (red bar) relative to sham (blue bar). Panel (b): Modulation of beta power in the post-stimulation stage across stimulation conditions with beta power at the baseline as covariate. The $x$-axis shows the 1 st, 2 nd, and 3rd quartile of the covariate, respectively $-14.6,-14.3,-13.9$ power units, while the $y$-axis represents beta power at the post-stimulation stage. At high levels of beta power at the baseline (i.e., the 3rd quartile), a significant reduction of beta power in the post-stimulation stage was found only for Conventional tDCS (green bar) relative to both HD-tDCS and sham. Panel (c): Response times (RTs) modulation across stimulation conditions with beta power at the baseline stage as covariate. In the $\mathrm{x}$-axis, the 1st, 2nd, and 3rd quartile of the covariate, namely -14.6, -14.3,-14 power units, while in the $y$-axis, RTs at the Finger Tapping Task performed during the post-stimulation stage. At low levels of beta power in the baseline stage (i.e., the 1st quartile), results show a significant reduction of RTs in HD-tDCS relative to sham. Note: * $(p<.05)$; $(p=.068)$. 


\section{Conventional tDCS}

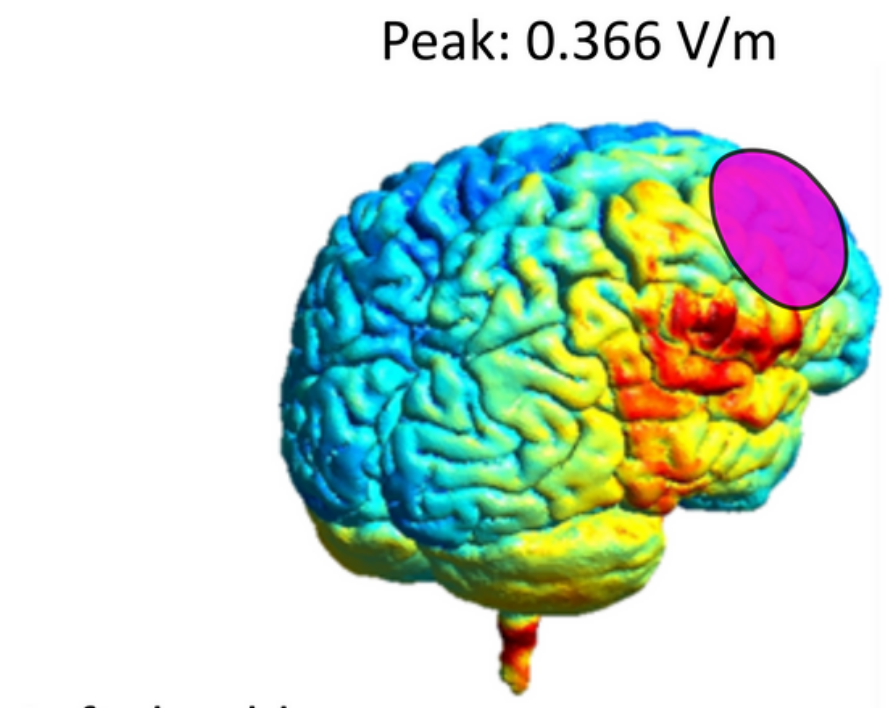

\section{High-definition tDCS}

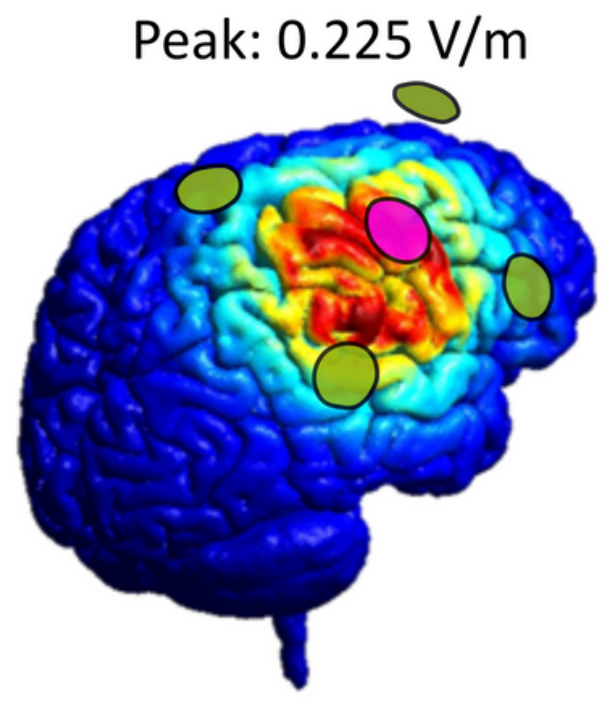

\section{Left shoulder}
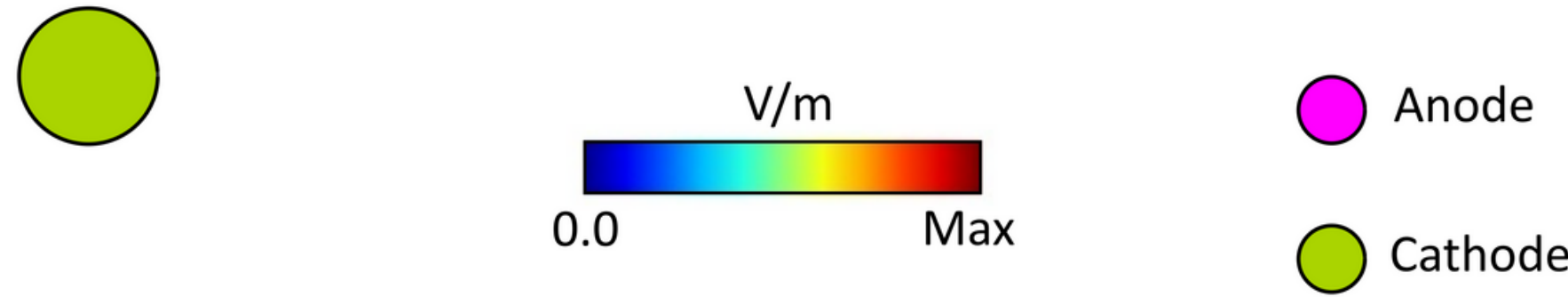

\section{Figure 2}

Conventional vs. HD-tDCS simulations. Finite element method (FEM) simulations showing the electric fields of Conventional and HD-tDCS montages, both estimated by SimNIBS 48. In the image on the left, the electric field of Conventional tDCS (anode: C4; cathode: left shoulder). On the right, the electric field of HD-tDCS (anode: C4; cathodes: FC2, FC6, CP2, CP6). As shown, the electric field elicited by HDtDCS is much more focal than Conventional tDCS where the current is more spread.

展

Figure 3

Experimental procedure, tDCS montages, and task. Panel (a): Representation of the experimental procedure. Each participant took part to three experimental sessions that were separated by at least 6 days one another and where participants received Conventional tDCS, HDtDCS, and sham. Blocks of the Finger Tapping Task were administered at the baseline, during the stimulation stage and at 5 minutes poststimulation. Resting-state EEG was recorded at the baseline as well as after the stimulation stage. At the end of the experimental session the Purdue Pegboard Test was administered. Panel (b): Head diagrams of Conventional and HD-tDCS electrode montages embedded in the EEG cap, which contained a total of 20 recording electrodes. In Conventional tDCS, the anode was placed on C4 and the cathode on the left shoulder. In HD-tDCS, the anode was placed on C4 and the cathodes on FC2, FC6, CP2, CP6. Panel (c): Illustration of the Finger Tapping Task. Each trial started with the onset of a fixation point for $500 \mathrm{~ms}$. Then, a sound was simultaneously presented with the string. Participants were asked to digit the same string 12 times in a row, after which a new string was presented. Note: Finger Tapping Task (FTT); Purdue Pegboard Test (PPT).

\section{Supplementary Files}

This is a list of supplementary files associated with this preprint. Click to download. 
- supplementarymaterial.pdf

Page 13/13 